\title{
The blinking eye: A window into tinnitus
}

Lars Rogenmoser ${ }^{1,2^{*}}$, Paweł Kuśmierek ${ }^{1}$, Denis Archakov ${ }^{1}$, Josef P. Rauschecker ${ }^{1}$

${ }^{1}$ Department of Neuroscience, Georgetown University Medical Center, 3970 Reservoir Road, Washington DC 20057, USA

${ }^{2}$ Department of Medicine, University of Fribourg, Chemin du Musée 8, 1700, Fribourg, Switzerland

*Corresponding author. E-mail address: lars.rogenmoser@unifr.ch (L. Rogenmoser) 


\begin{abstract}
Tinnitus is a prevalent untreatable audiological disorder, charaterized by the perception of phantom sound. Despite longstanding research with animals, its underlying pathophysiology remains poorly understood. The obstacle in progressing in the field may lie in both the current species of choice and the available measurements of tinnitus for animals. To provide fresh impetus, we developed a novel tinnitusverification technique applicable to rhesus monkeys. Tinnitus was induced via salicylate administration in two rhesus monkeys, and was confirmed by applying a specific eye-blinking procedure: Blinks, as monitored using EMG, were triggered via puffs of air towards the cheek, and their modulation was studied as a function of preceding tones with various frequency and intensity conditions. The advantage in using a tactile reflex-inducing stimulus lies in its non-auditory modality, bypassing potential confounding factors of hearing loss and hyperacusis. Interference effects on the blink modulation pattern was interpreted as tinnitus, and the interfering frequency of the preceding interfering tone as tinnitus frequency. A crossvalidation in a sample of tinnitus patients revealed interfering effects of the preceding tone at the specific frequency range corresponding to their own tinnitus frequency, as independently determined by audiologists. This interference effect increased as a function of their individual tinnitus loudness. In conclusion, the present work demonstrates a considerable transferability of our newly established tinnitusverification technique from non-human primates to human tinnitus patients.
\end{abstract}




\section{Introduction}

Tinnitus is a widespread medical condition with the core symptom of hearing phantom noise (Lockwood et al., 2002). It affects about $15 \%$ of the general and up to $45 \%$ of the older population (Chang et al., 2019; Henry et al., 2005; Jafari et al., 2019), debilitating the patient sometimes with accompanying cognitive-affective and somatic symptoms (Neff et al., 2021; Salazar et al., 2019; Shargorodsky et al., 2010). In worst cases, this condition may even lead to suicidal tendencies (Szibor et al., 2019). While the phantom noise is usually perceived in the ears, it is rather generated by the brain (Berliner et al., 1992; Fahy et al., 2002; Magliulo et al., 2000). Generally, tinnitus is initiated by hearing loss caused by damage of cochlear hair cells together with ribbon synapses and/or spiral ganglion cells (Bauer et al., 2007; Kaltenbach et al., 2004; Singer et al., 2013). This aberration may result from genetic abnormalities, injury, exposure to loud noise or ototoxic substances and/or from degenerative processes associated with aging (Cunningham and Tucci, 2017). The partial loss of peripheral auditory input leads to an imbalance that triggers plastic compensatory mechanisms within the auditory system. As a result, phantom noise is introduced into auditory perception, i.e., tinnitus emerges. As such, tinnitus can be described as a maladaptive plasticity effect (Rauschecker, 1999; Shore et al., 2016), which is documented as tonotopic remapping (Dietrich et al., 2001; Mühlnickel et al., 1998; Wienbruch et al., 2006) or more broadly as change, mainly of gain, in the cellular firing rate or in neural synchrony throughout the auditory pathways (Eggermont, 2015; Noreña, 2011). However, an impaired auditory system alone is not solely responsible for the pathophysiology of tinnitus, and it hardly determines its course or the impact of the suffering on the patient (De Ridder et al., 2011; Leaver et al., 2012). A large body of evidence suggests that in addition to the auditory system particular perception-unrelated brain structures and networks are involved in the pathophysiology as well. Human and animal studies identified cerebellar structures contributing to the generation of tinnitus (Bauer et al., 2013; Brozoski et al., 2017; Chen et al., 2015; Mennink et al., 2018). Tinnitus-related changes have further been identified in limbic (i.e., amygdala, hippocampus, parahippocampus, nucleus accumbens, striatum) and paralimbic (i.e., insula, cingulate) regions that likely account for the affective, attentive and 
mnemonic processes implicated in the pathophysiology (Burton et al., 2012; De Ridder et al., 2006; Landgrebe et al., 2009; Leaver et al., 2011; Mühlau et al., 2006; Schecklmann et al., 2013; Schmidt et al., 2018; Vanneste et al., 2010). These changes at the paralimbic and limbic level likely contribute to the mechanisms underlying the conscious awareness and appraisal of the phantom noise. Finally, human imaging studies linked a wide range of cortical non-auditory regions (i.e., temporo-parietal and prefrontal) to impairment of top-down control implicated in tinnitus (Giraud et al., 1999; Leaver et al., 2012, 2011; Paraskevopoulos et al., 2019; Schmidt et al., 2018; Seydell-Greenwald et al., 2012; Vanneste et al., 2010). Multiple studies have revealed that the ventromedial prefrontal cortex (vmPFC) undergoes significant volume loss in tinnitus patients (Leaver et al., 2012, 2011; Mühlau et al., 2006). This morphological change likely disables top-down gating and inhibition normally exerted by vmPFC on auditory centers, thus preventing normally available suppression of phantom noise (Rauschecker et al., 2015, 2010).

Despite the usage of various animal models over the last three decades, the exact mechanisms causing and driving tinnitus are still far from understood, as to this day we are still unable to accurately predict from the outcome of hearing damage the profile, let alone the course or even the emergence rate of tinnitus. Small mammals, such as the cat and various rodent species (i.e., mouse, rat, gerbil, hamster, guinea pig, and chinchilla) are currently used as subjects in tinnitus studies (Noreña and Eggermont, 2003; von der Behrens, 2014). However, developing the appropriate tinnitus-verification technique turned out to be challenging (Brozoski and Bauer, 2016). Currently, the most commonly used one is a technique referred to as Gap-Prepulse Inhibition of the Acoustic Startle (GPIAS) (Turner et al., 2006). In this procedure, the natural Acoustic Startle Reflex (ASR) is experimentally modulated by preceding the startle burst with short gaps embedded in an auditory noise stimulus. Analogous to the mechanism of Prepulse Inhibition (Koch, 1999; Swerdlow et al., 1999), short gaps embedded in a background of ongoing noise are known to inhibit the ASR as well (Ison, 1982). The principle of the GPIAS argues that it is possible to objectively measure the presence of tinnitus by configuring the ongoing background noise to mimic the phantom (tinnitus) noise: If the background noise mimics tinnitus, the phantom sound should "fill in" the gaps and make them less 
perceptible, consequently reducing the inhibition of the ASR and thus providing an objective marker of tinnitus. However, the validity of the GPIAS technique has been increasingly criticized given that not all findings could be explained with the "filling-in-the-gap" argument and, most importantly, their results failed to translate to actual tinnitus patients (An et al., 2014; Boyen et al., 2015; Campolo et al., 2013; Fournier and Hébert, 2013; Galazyuk and Hébert, 2015; Ku et al., 2017; Mahmoudian et al., 2013).

In hope of providing fresh impetus, we pilot tested a novel tinnitus-verification technique on a species more closely related to us: the rhesus monkey (Macaca mulatta), a nonhuman primate (NHP). We used a specific Eye-Blinking (EB) paradigm to assess salicylate-induced tinnitus in two NHPs and actual tinnitus in a sample of human patients. In this paradigm, we deliver puffs of air towards the cheek that either are preceded by tones or not. Given that inputs of polysensory sources are known to modulate the excitability triggering the blink reflex (Miwa et al., 1998; Valls-Sole, 2012), our model hypothesizes analogous to the GPIAS that tinnitus interferes with the effect of the tone on the puff-evoked blink response if its properties match the phantom sound.

\section{Methods}

All animal and human procedures were approved by the Institutional Animal Care and Use Committee and the Institutional Review Board of Georgetown University, respectively. All human participants gave written informed consent before participating in this study.

\subsection{Animals}

Two male NHPs (monkey C and monkey S; 14 and 7 years of age, respectively) were used in this study, each implanted with a headpost (Applied Prototype) secured to the skull with ceramic screws (Thomas Recording) or titanium cortical bone screws (Veterinary Orthopedic Implants), plastic strips, and bone cement (Osteobond, Zimmer). All surgical procedures were conducted under aseptic conditions using isoflurane anesthesia (1-2\%) following pre-anesthetic medication with ketamine (10-15 mg/kg) and midazolam $(0.1-0.5 \mathrm{mg} / \mathrm{kg})$, with postoperative analgesic and prophylactic antibiotic treatment. Prior data 
acquisition, the NHPs were trained to lie in sphinx position restrained by a primate chair (Applied Prototype) while being head-fixed. Data acquisition took place with this position in a sound-attenuated chamber (IAC, Bronx, NY).

\subsubsection{Animal protocol}

Data acquisition involved Eye-Blinking (EB) and Auditory Brainstem Response (ABR) recordings, which were performed in multiple daily sessions. Per session, ABRs were recorded in order to determine the hearing thresholds, which were used to calibrate the subsequent EB setup. Three blocks each of 6 sessions were carried out. The first block constituted baseline recordings. In the second block, salicylate was administered in order to induce tinnitus. Salicylate $(200 \mathrm{mg} / \mathrm{kg}$ ) in form of Aspirin powder (Vedco, Inc) mixed with food (e.g., yoghurt, honey, peanut butter) was administered to the animal every evening (7 pm) preceding the next day of $\mathrm{ABR}$ and $\mathrm{EB}$ recording. In total, the drug administration was done 7 days in a row, whereby data acquisition took place on the second treatment day and the five subsequent ones. The third block constituted follow-up recordings, and it was carried out 2-3 weeks after the final salicylate dose was administered.

\subsubsection{Auditory Brainstem Response (ABR) recordings}

ABR recordings took place early in the morning (5-8 a.m.), when the NHP was still sleepy. The chamber was kept dimmed in order to maintain the NHP's somnolent state, which was monitored via CCTV camera. Three silver-chloride $(\mathrm{Ag} / \mathrm{AgCl})$ cup electrodes (ELT-4830, EEG Sales LLC) were attached at the center of the supraorbital ridge (non-inverting input) and at both mastoids (inverting input and ground, respectively) using electrode gel (101, Biopac systems) together with adhesive tape. Auditory stimuli consisted of 1/3-octave band-passed noise bursts centered at 1, 2, 4, 8 and $16 \mathrm{kHz}$ with respective lengths based on the 2-1-2 cycle (i.e., rise-plateau-fall) principle (Stapells and Oates, 1997). They were delivered in alternating polarities (i.e., condensation and rarefaction) at a rate of 25/s via a loudspeaker (MSP3; Yamaha) which was placed about $20 \mathrm{~cm}$ in front of the animal. Stimuli-evoked responses were amplified 
(gain 100,000x) and band-pass filtered (100-1,500 Hz, CED 1902). The sound pressure level (SPL) was set at $75 \mathrm{~dB}$, and was then systematically decreased in 15-dB steps (CED 3505) until the evoked responses were no longer visually observable in averaged signal. The SPL was then increased in 3-dB steps until the responses were visually observable again, determining the hearing threshold at a given frequency. Each stimulus was repeated a minimum of 2000 times to obtain reliable average responses.

\subsubsection{Eye-Blinking (EB) recordings}

Blinks were monitored by recording the electromyography (EMG) under the right eye in response to airpuffs delivered towards the left cheek. Three $\mathrm{Ag} / \mathrm{AgCl}$ cup electrodes (ELT-4830, EEG Sales LLC) were attached according to a standard human protocol (Blumenthal et al., 2005), namely two below the orbicularis oculi muscle under the right eye (inverting and non-inverting input) and one on the forehead (ground). Electrode gel (101, Biopac systems) and adhesive tape were used to ensure attachment. The airpuffs were delivered with a pressure of $30 \mathrm{psi}$ and duration of $10 \mathrm{~ms}$. They were either delivered alone (probability rate of 0.25 ) or with a tone (probability rate of 0.75 ), preceding the puffs with $50 \mathrm{~ms}$. The tones consisted of 1/3-octave band noises centered at 1, 2, 4, 8 and $16 \mathrm{kHz}$ with a length of $20 \mathrm{~ms}$ (Blackman fade-in/outs of $5 \mathrm{~ms}$ ). Per frequency condition, the tones were presented at 3 different sensation levels (SL; each with a probability rate of 0.25 ), which were threshold-adjusted based on the ABR outcomes. The threshold-adjusted intensities were 30,6 and $-6 \mathrm{~dB}$ SL. The $30 \mathrm{~dB}$ SL provided a condition of clear audibility, the $6 \mathrm{~dB}$ SL one overlapping with the range of expected tinnitus loudness (Fowler, 1938; Graham and Newby, 1962; Henry, 2016; Reed, 1960; Roeser and Price, 1980) and the -6 dB SL a condition of inaudibility. The three sensation levels together with the "no-tone" condition were randomized within one frequency block. Each condition was presented 12 times, and the inter-trial interval was jittered between 5 and $25 \mathrm{~s}$. The order of the blocks was randomized. The tones were delivered via loudspeaker (MSP3; Yamaha), which was placed about $20 \mathrm{~cm}$ on front of the animal. The airpuffs were controlled via transistortransistor logic signals using a solenoid valve (\#003-0111-900, Parker Hannifin model), and were delivered from an air tank through a nozzle pointed towards the animal's left cheek. Puff-evoked EMG responses 
were amplified (gain 1,000x) band-pass filtered (10-500 Hz, CED 1902).

\subsection{Humans}

In order to determine transferability of the tinnitus-verification technique, the same ABR and EB recordings were performed in samples of tinnitus patients and matched control subjects (each $N=11)$. The stimulus material, filter settings and presentation protocols were identical to the ones used for the NHPs. The two human samples were comparable regarding age (patients: $M=49, S D=16.91$, range 20-75; controls: $M=46, S D=14.66$, range $\left.26-66 ; t_{19.6}=0.44, P=0.66\right)$ and the distribution of the sexes (9 male patients and 5 male controls; Fisher's Exact test, $P=0.18$ ). The patients perceived their tinnitus at an average frequency of $8.73 \mathrm{kHz}(S D=2$, range 6-14 kHz) with an average loudness of $10.18 \mathrm{~dB}$ SL $(S D=$ 6.23, range 2-20 dB SL), as independently revealed by standardized frequency and loudness matching by certified audiologists at MedStar Georgetown University Hospital. The impact of the tinnitus on their daily living, as measured by the Tinnitus Handicap Inventory (THI) (Newman et al., 1996), varied among patients, ranging from slight to catastrophic $(M=.21 .45, S D=89$, range 4-86 THI scores). The patients' individual characteristics on tinnitus are detailed in Table 1.

The participants received two appointments: the first one for determining the hearing thresholds and the second one for EB recordings. All testing took place in a sound-attenuated chamber (IAC, Bronx, NY). Before performing the ABR recording, the participants underwent a behavioral audiogram with the specific ABR stimuli. The participants gave feedback by raising their hand when they were able to hear the presented stimuli, which were manipulated in the same stair-wise manner, determining the behavioral hearing thresholds at a given frequency. During ABR recordings, the chamber was kept dimmed. The participants lied on a stretcher with their eyes closed, and were encouraged to take a nap. The stimuli were delivered binaurally via Sennheiser HD200 headphones. The EB recordings took place in a sitting relaxed position. Airpuffs were delivered via a nozzle pointing towards the left cheek that was attached to the Sennheiser HD200 headphones. The headphones delivered the preceding tones. For both the ABR and EB recordings, three $\mathrm{Ag} / \mathrm{AgCl}$ cup electrodes (ELT-4830, EEG Sales LLC) were applied using the same 
configuration as for the NHPs.

\subsection{Stimuli presentation and data recording}

In both humans and NHPs, stimuli presentation and data recording were controlled by a Power1401 laboratory interface (CED) together with the Spike2 software. All SPLs were calibrated using a sound level meter (Bruel \& Kjaer 2235). Electrode impedances were kept below $5 \mathrm{k} \Omega$, as monitored via EL-Check (Biopac systems). In the NHPs, the surface of the recording spots was shaved and cleaned using alcohol wipes. In both, non-conductive skin cells were removed by gently rubbing the spots with abrasive pads (ELPAD, Biopac systems).

\subsection{Data analyses}

Data processing and analyzes were conducted using Spike2 (v. 9.10) and Matlab (v. R2018b). The raw EMG data for each subject were rectified. Per airpuff response, epochs were created, ranging from -10 to $170 \mathrm{~ms}$ after puff exposure. A baseline correction relative to the "-10 to $0 \mathrm{~ms}$ " segment was applied. Condition-wise, these epochs were averaged. The maximal values were extracted from the averaged puffevoked responses. Within each frequency block (i.e., 1, 2, 4, 8, $16 \mathrm{kHz}$ ), the blink modulation was calculated by subtracting the maximal value pertaining to the "no-tone" condition respectively from the maximal values pertaining to the condition with the tone of each intensity level (i.e., $30,6,-6 \mathrm{~dB}$ SL). These difference scores were converted into absolute values, and then logarithmically transformed. In sum, the formula for computing the blink modulation as a function of the preceding tone was:

$$
\text { Blink modulation }=\log \left(\mid \text { Puff }_{\text {tone }}-\text { Puff }_{\text {no-tone }} \mid\right)
$$

These scores were imported into the SPSS software (v. 28) for statistical analyses. To explore for interference effects of tinnitus, the scores were frequency-wise subjected to analyses of variance (ANOVA) with the 3-level within-factor(s) "intensity" (i.e., 30, 6, -6 dB SL) and either "recording block" (i.e., baseline, salicylate, follow-up) for NHPs or the 2-level between-factor "group" (i.e., patients, controls) for humans. For each NHP, the statistical analyzes were conducted separately, whereby the session days served 
as the unit of observation.

\section{Results}

\subsection{NHP findings}

The individual ABR audiograms revealed by both NHPs across the recording days are depicted in Fig 1. Apparent in both NHPs are the elevated hearing thresholds mostly at the upper frequency range in response to the salicylate administration and its reversal in the follow-up days.

The grand-averaged EMG blink responses for each NHP to all conditions of frequency, intensity and recording block can be found in Fig. S1 and Fig S2. Their individual blink modulation scores are depicted in Fig. 2, and the ANOVA results are detailed in Table 2. Both NHPs exhibited highly significant main effects of the factor "intensity" across all frequency conditions $(P \leq 0.001)$. An interference on the NHPs' eye-blinking behavior was apparent at the $16 \mathrm{kHz}$ condition, as revealed by the significant interaction effects between the factors "intensity" and "recording block" $(\mathrm{C}: P<0.005$; S: $P<0.05)$. These interaction effects were due to the specific recording block with salicylate administration, as in comparison to the baseline and follow-up scores, the blink modulation under the influence of salicylate varied differently as a function of the intensity level. Particularly, the puff-evoked blinks preceded by the $16 \mathrm{kHz}$ tones within the loudness range of tinnitus (i.e., $6 \mathrm{~dB} \mathrm{SL}$ ) did not modulate more strongly than the ones preceded by the inaudible (i.e., $-6 \mathrm{~dB}$ SL) tones of the same frequency $\left(\mathrm{C}: t_{5}=0.41, P=0.70 ; \mathrm{S}: t_{5}=2.15\right.$, $P=0.08)$, whereas the blinks recorded in the baseline and follow-up blocks at the same frequency did (C and $\left.\mathrm{S}: t_{5}>2.5, P<0.05\right)$. In addition to these interaction effects, monkey $\mathrm{C}$ demonstrated significant main effects of the factor "recording block", mainly at the upper frequency range (i.e., 4-16 kHz).

\subsection{Human findings}

The ABR and behavioral audiograms of both samples are depicted in Fig. 3. Both samples were comparable regarding their hearing profile, as revealed by a mixed ANOVA with "group" (i.e., patients, controls) as between-factor and "frequency" (i.e., 1, 2, 4, 8, $16 \mathrm{kHz}$ ) and "audiogram" (i.e., ABR, behavioral 
testing $)$ as within factors. Indeed, the ANOVA revealed a highly significant effect of frequency $\left(F_{4,80}=\right.$ 19.27, $\left.P<3.9 \times 10^{-11}\right)$, none of audiogram $\left(F_{1,20}=0.04, P=0.85\right)$ and no significant group effect $\left(F_{1,20}=\right.$ $0.12, P=0.73$ ) or any significant group-interaction effects (group x frequency x audiogram: $F_{4,20}=0.61, P$ $=0.22$; group $\mathrm{x}$ frequency: $F_{4,20}=1.74, P=0.17$; group x audiogram: $F_{1,20}=0.47, P=0.88$ ). The thresholds across all frequencies correlated between the two audiometric methods (patients: $r_{11}>0.6, P<0.03$; controls: $\left.r_{11}>0.7, P<0.02\right)$.

The grand-averaged EMG blink responses for each group to all conditions of frequency and intensity can be found in Fig. S3. Their individual blink modulation scores are depicted in Fig. 4, and the ANOVA results are detailed in Table 3. The ANOVAs revealed highly significant main effects of the factor “intensity” across all frequency conditions $\left(P<3.9 \times 10^{-5}\right)$. An interference on the eye-blinking behavior was spotted only at the $8 \mathrm{kHz}$ condition, as revealed by the highly significant group and group-interaction effects $(P<0.01)$. The frequency of the preceding tones within this specific condition largely overlapped with the frequency range at which the patients perceived their tinnitus $(M=8.73, S D=2 \mathrm{kHz}$; see Table 1). In comparison to the controls, the patients exhibited overall reduced blink modulations (i.e., group effect) and additionally an altered blink modulation pattern (i.e., group-interaction effect). This pattern was reflected by the suspension of stronger puff-evoked blink modulations when preceded by the $8 \mathrm{kHz}$-tones set within the loudness range of tinnitus (i.e., $6 \mathrm{~dB}$ ) than when preceded by the inaudible (i.e., $-6 \mathrm{~dB}$ ) tones of the same frequency $\left(t_{10}=0.03, P=0.98\right)$. In contrast, the puff-evoked blink modulations of the controls preceding the $8 \mathrm{kHz}$-tones set at $6 \mathrm{~dB}$ SL overshot the ones preceding the tones set at $-6 \mathrm{~dB}$ of the same frequency $\left(t_{10}=2.90, P=0.02\right)$. Finally, the patients demonstrated a negative correlation between tinnitus loudness and the puff-evoked blink modulation preceded by the $8 \mathrm{kHz}$-tone set at $6 \mathrm{~dB}$ SL $\left(r_{11}=-0.59, P=\right.$ 0.03; see Fig. 5). No other significant correlations were found between tinnitus loudness and the blink modulation of any condition constellation $\left(r_{11}< \pm 0.43, P>0.07\right)$.

\section{Discussion}

In the present work, we pilot tested a novel tinnitus-verification technique applicable to the NHP 
as an animal model of tinnitus. In both our NHPs, the procedure was able to identify an interference effect of tinnitus on the eye-blinking behavior in response to the salicylate induction. This interference effect was characterized by altered blink modulations in response to the upper frequency conditions; in particular by the suspension of the otherwise increased blink modulations when the airpuff was preceded by the $16 \mathrm{kHz}-$ tone set at $6 \mathrm{~dB}$ SL, the expected range of tinnitus loudness (Fowler, 1938; Graham and Newby, 1962; Henry, 2016; Reed, 1960; Roeser and Price, 1980). Given the specificity of the revealed interference effects unfolding within this particular frequency and loudness range, we inferred tinnitus-matching properties of this particular tone, thus determining the presence of tinnitus at that particular frequency, namely $16 \mathrm{kHz}$. This finding is in line with previous human and animal studies (Bauer et al., 1999; Day et al., 1989; Janssen et al., 2000; Liu and Chen, 2015; Martel et al., 2019; Mccabe and Dey, 1965; Stolzberg et al., 2011; Yang et al., 2007), reporting salicylate-induced tinnitus at the higher frequency range (Cazals, 2000; Eggermont, 2015). Our cross-validation in human samples revealed a comparable interference effect at $8 \mathrm{kHz}$, corresponding to the frequency range independently determined by audiologists. Within this specific frequency condition, the patients not only exhibited the suspension of the otherwise increased blink modulations but additionally showed an interference as a function of their tinnitus loudness. In conclusion, the present work suggests considerable transferability of our newly established EB-setup to human tinnitus patients. Fig. 6 comparatively depicts the outcome of the pilot test revealed from both humans and NHPs.

\subsection{The NHP as promising animal model of tinnitus}

Over the last few decades, rodent models were able to gain important insights into the cellular and molecular mechanisms contributing to the plasticity and metaplasticity that induce or drive tinnitus (Haider et al., 2018; Knipper et al., 2020; Shore and Wu, 2019). However, these animal models limited their scope rather to peripheral and brain structures throughout the auditory pathways. Rodents are hardly comparable to humans regarding particular medial prefrontal brain structures (i.e., BA 13b and 14r) (Öngür and Price, 2000; Preuss, 1995), which are undoubtedly vital for the full understanding of tinnitus (Jastreboff, 1990; Leaver et al., 2012, 2011; Rauschecker et al., 2015, 2010; Seydell-Greenwald et al., 2012), and may 
potentially expand the treatment possibilities when targeting them with sophisticated neuromodulation devices (Rauschecker et al., 2010). The NHPs possess more established and human-like prefrontal structures than rodents or carnivores, thus an NHP-based tinnitus model promises to capture the disorder more adequately. In addition, the NHP offers ecological validity unparalleled by other animal models not only due to critical similarities to humans in terms of neuroanatomy but also in terms of hearing range and lifespan (Recanzone, 2018). NHPs hold great potential for clinical translation to humans, as demonstrated across multiple fields in biomedical sciences, particularly in aging-related topics (Capitanio and Emborg, 2008). The usage of NHPs permit experimental approaches that can be applied to humans with little modification. In the present work, we were able to apply very similar recording protocols in both NHPs and humans, ensuring between-species translatability. Specifically, we monitored the EB behavior in NHPs as well in humans with non-invasive EMG. By contrast, in rodent studies the ASR is commonly recorded using startle chambers, bearing far less translational value.

\subsection{Advantage of tactile reflex-inducing stimuli for tinnitus-verification}

Instead of relying on the ASR for tinnitus-verification as in the previous GPIAS studies, we used a tactile airpuff to trigger the blink reflex. In comparison to the ASR, the airpuff-evoked blink reflex is significantly more reliable, with a response rate of virtually $100 \%$ (Haerich, 1998). This reliability was confirmed in our findings, as in both humans and NHPs the blink modulation was robust across all frequency conditions, with intensity effects of moderate to large sizes (see Table 2 and 3). GPIAS studies not only bear the problem of having to deal with "none-responder" animals and/or trials during data processing but also often find altered amplitudes of the ASR after tinnitus induction. A reduced ASR amplitude after tinnitus induction has been attributed to hearing loss and an augmented ASR amplitude to hyperacusis, respectively (Chen et al., 2013, 2015; Lobarinas et al., 2013; Longenecker and Galazyuk, 2011), both conditions confounding with tinnitus effects and thus impairing the tinnitus-verification performance. The tactile airpuff as a reflex-inducing stimulus bypasses this confounding factor due to its non-auditory modality. Furthermore, the airpuff is less aversive than the ASR-related sound burst (Lissek 
et al., 2005). This aspect is crucial given that tinnitus is often comorbid with hyperacusis (Hayes et al., 2014). The hyperacusis-related painful sensations in response to the loud sound bursts may additionally interfere with the diagnostic power of the verification technique, as stress is known to interact with tinnitus (Alpini and Cesarani, 2006; Kim et al., 2021; Schmitt et al., 2000).

\subsection{Reflex inhibition and facilitation}

Our newly established EB-setup revealed both facilitation and inhibition of the airpuff-evoked blink reflex, a phenomenon often observed in GIAPS studies as well (Longenecker et al., 2018). As discussed by Longenecker et al., 2018, the reason for this dichotomy is not yet fully understood. Nevertheless, both systematic modulation types reflect most likely a contribution of the encoding of the reflex-preceding event. We decided to treat both types as such, and to study the modulation regardless of its direction. For this, we extracted the absolute values of the differences within a given frequency block between the puff-evoked responses with preceding tones and the puff-evoked responses without preceding tones. To counteract large variabilities across the scores, we additionally performed a logarithmical transformation, a technique previously suggested in analyzing eye-blinking behavior (Ornitz et al., 1986). Indeed, particular modified ratios for correcting this "direction problem" and sophisticated data-processing approaches for generally reducing variability in ASR data have been suggested in the past (Grimsley et al., 2015; Longenecker et al., 2018; Longenecker and Galazyuk, 2012). To the best of our knowledge, our correction formula has not been applied before.

\subsection{Future directions}

In our work, we chose to induce tinnitus in NHPs via salicylate administration due to its reversibility, reliability, and rapid effect. Salicylate administration as an induction-method models tinnitus fairly well, as the substance enters the cochlear perilymph and cerebrospinal fluid, unfolding the tinnituscontributing effects at peripheral and the key brain structures involved in tinnitus perception (Stolzberg et al., 2012). However, the degree to which the underlying mechanisms overlap with the ones underlying 
tinnitus of other etiologies are still debated (Eggermont, 2016; Lanaia et al., 2021). The cross-validation performed on our tinnitus patients suggests that the EB-setup is capable of also detecting tinnitus of the more typical etiologies, such as noise exposure or perhaps age. In the context of the NHP as an animal model, an investigation of the latter would be uniquely fruitful. Under captivity, some NHPs manage to live long enough to naturally develop age-related hearing loss (Engle et al., 2014, 2013; Gray et al., 2014a, 2014b, 2013; Ng et al., 2015), which potentially may involve tinnitus. Identifying geriatric NHPs with tinnitus might constitute a future milestone with great impetus for tinnitus research and treatment development. Given that our verification technique is non-invasive and relatively free from aversion (Lissek et al., 2005), it may be considered refined especially for sensitive populations such as elderly subjects with hearing issues (Haerich, 1998). This aspect together with the fact that the technique relies solely on a reflex and consequently does not require instrumental conditioning or animal training allows rapid testing of larger colonies.

\section{Acknowledgements}

This work was supported by the Swiss National Science Foundation (168587, 177744, 186636, 195992 to LR), the Toulmin Pilot Award (JPR and LR), and the Tinnitus Research Consortium (JPR). We thank Dr. Robin Tucker for providing veterinary care to the NHPs.

\section{References}

Alpini, D., Cesarani, A., 2006. Tinnitus as an alarm bell: Stress reaction tinnitus model. ORL.

An, Y.H., Jin, S.Y., Yoon, S.W., Shim, H.J., 2014. The effects of unilateral tinnitus on auditory temporal resolution: Gaps-in-noise performance. Korean J. Audiol. 18, 119-125.

Bauer, C.A., Brozoski, T.J., Myers, K., 2007. Primary afferent dendrite degeneration as a cause of tinnitus. J. Neurosci. Res. 85, 1489-1498. 
Bauer, C.A., Brozoski, T.J., Rojas, R., Boley, J., Wyder, M., 1999. Behavioral model of chronic tinnitus in rats. Otolaryngol. - Head Neck Surg. 121, 457-462.

Bauer, C.A., Kurt, W., Sybert, L.T., Brozoski, T.J., 2013. The cerebellum as a novel tinnitus generator. Hear. Res. 295, 130-139.

Berliner, K.I., Shelton, C., Hitselberger, W.E., Luxford, W.M., 1992. Acoustic tumors: Effect of surgical removal on tinnitus. Otol. Neurotol. 13, 13-17.

Blumenthal, T.D., Cuthbert, B.N., Filion, D.L., Hackley, S., Lipp, O. V., Van Boxtel, A., 2005. Committee report: Guidelines for human startle eyeblink electromyographic studies. Psychophysiology 42, 1-15.

Boyen, K., Başkent, D., Van Dijk, P., 2015. The gap detection test: Can it be used to diagnose tinnitus? Ear Hear. 36, e138-e145.

Brozoski, T., Brozoski, D., Wisner, K., Bauer, C., 2017. Chronic tinnitus and unipolar brush cell alterations in the cerebellum and dorsal cochlear nucleus. Hear. Res. 350, 139-151.

Brozoski, T.J., Bauer, C.A., 2016. Animal models of tinnitus. Hear. Res.

Burton, H., Wineland, A., Bhattacharya, M., Nicklaus, J., Garcia, K.S., Piccirillo, J.F., 2012. Altered networks in bothersome tinnitus: A functional connectivity study. BMC Neurosci. 13.

Campolo, J., Lobarinas, E., Salvi, R., 2013. Does tinnitus «fill in» the silent gaps? Noise Heal. 15, 398405.

Capitanio, J.P., Emborg, M.E., 2008. Contributions of non-human primates to neuroscience research. The Lancet.

Cazals, Y., 2000. Auditory sensori-neural alterations induced by salicylate. Prog. Neurobiol.

Chang, N.C., Dai, C.Y., Lin, W.Y., Yang, H.L., Wang, H.M., Chien, C.Y., Ho, K.Y., 2019. Prevalence of 
persistent tinnitus and dizziness in an elderly population in southern taiwan. J Int Adv Otol 15, 99105.

Chen, G., Lee, C., Sandridge, S.A., Butler, H.M., Manzoor, N.F., Kaltenbach, J.A., 2013. Behavioral evidence for possible simultaneous induction of hyperacusis and tinnitus following intense sound exposure. J. Assoc. Res. Otolaryngol. 14, 413-424.

Chen, Y.C., Li, X., Liu, L., Wang, J., Lu, C.Q., Yang, M., Jiao, Y., Zang, F.C., Radziwon, K., Chen, G. Di, Sun, W., Muthaiah, V.P.K., Salvi, R., Teng, G.J., 2015. Tinnitus and hyperacusis involve hyperactivity and enhanced connectivity in auditory-limbic-arousal-cerebellar network. eLife 4.

Cunningham, L.L., Tucci, D.L., 2017. Hearing loss in adults. N. Engl. J. Med. 377, 2465-2473.

Day, R., Graham, G., Bieri, D., Brown, M., Cairns, D., Harris, G., Hounsell, J., Platt- Hepworth, S., Reeve, R., Sambrook, P., et al, 1989. Concentration-response relationships for salicylate-induced ototoxicity in normal volunteers. Br. J. Clin. Pharmacol. 28, 695-702.

De Ridder, D., Elgoyhen, A.B., Romo, R., Langguth, B., 2011. Phantom percepts: Tinnitus and pain as persisting aversive memory networks. Proc. Natl. Acad. Sci.

De Ridder, D., Fransen, H., Francois, O., Sunaert, S., Kovacs, S., Van De Heyning, P., 2006. Amygdalohippocampal involvement in tinnitus and auditory memory, in: Acta Oto-Laryngol. pp. $50-53$.

Dietrich, V., Nieschalk, M., Stoll, W., Rajan, R., Pantev, C., 2001. Cortical reorganization in patients with high frequency cochlear hearing loss. Hear. Res. 158, 95-101.

Eggermont, J.J., 2016. Can animal models contribute to understanding tinnitus heterogeneity in humans? Front. Aging Neurosci.

Eggermont, J.J., 2015. The auditory cortex and tinnitus - a review of animal and human studies. Eur. J. Neurosci. 41, 665-676. 
Engle, J.R., Gray, D.T., Turner, H., Udell, J.B., Recanzone, G.H., 2014. Age-related neurochemical changes in the rhesus macaque inferior colliculus. Front. Aging Neurosci. 6, 73.

Engle, J.R., Tinling, S., Recanzone, G.H., 2013. Age-Related Hearing Loss in Rhesus Monkeys Is Correlated with Cochlear Histopathologies. PLoS ONE 8, e55092.

Fahy, C., Nikolopoulos, T.P., O’Donoghue, G.M., 2002. Acoustic neuroma surgery and tinnitus. Eur. Arch. Oto-Rhino-Laryngology 259, 299-301.

Fournier, P., Hébert, S., 2013. Gap detection deficits in humans with tinnitus as assessed with the acoustic startle paradigm: Does tinnitus fill in the gap? Hear. Res. 295, 16-23.

Fowler, E.P., 1938. The use of threshold and louder sounds in clinical diagnosis and the prescribing of hearing aids. new methods for accurately determining the threshold for bone conduction and for measuring tinnitus and its effects on obstructive and neural deafness. Laryngoscope 48, 572-587.

Galazyuk, A., Hébert, S., 2015. Gap-prepulse inhibition of the acoustic startle reflex (GPIAS) for tinnitus assessment: Current status and future directions. Front. Neurol.

Giraud, A.L., Chéry-Croze, S., Fischer, G., Fischer, C., Vighetto, A., Grégoire, M.C., Lavenne, F., Collet, L., 1999. A selective imaging of tinnitus. NeuroReport 10.

Graham, J.T., Newby, H.A., 1962. Acoustical characteristics of tinnitus: An analysis. Arch. Otolaryngol. $75,162-167$.

Gray, D.T., Engle, J.R., Recanzone, G.H., 2014a. Age-related neurochemical changes in the rhesus macaque superior olivary complex. J. Cogn. Neurosci. 522, 573-591.

Gray, D.T., Engle, J.R., Recanzone, G.H., 2014b. Age-related neurochemical changes in the rhesus macaque cochlear nucleus. J Comp Neurol 522, 1527-1541.

Gray, D.T., Rudolph, M.L., Engle, J.R., Recanzone, G.H., 2013. Parvalbumin increases in the medial and 
lateral geniculate nuclei of aged rhesus macaques. Front Aging Neurosci 5, 69.

Grimsley, C.A., Longenecker, R.J., Rosen, M.J., Young, J.W., Grimsley, J.M., Galazyuk, A. V., 2015. An improved approach to separating startle data from noise. J. Neurosci. Methods 253, 206-217.

Haerich, P., 1998. Using airpuffs to elicit the human blink reflex. Behav. res. meth. instrum. comput. 30, $661-666$.

Haider, H.F., Bojić, T., Ribeiro, S.F., Paço, J., Hall, D.A., Szczepek, A.J., 2018. Pathophysiology of subjective tinnitus: Triggers and maintenance. Front. Hum. Neurosci.

Hayes, S.H., Radziwon, K.E., Stolzberg, D.J., Salvi, R.J., 2014. Behavioral models of tinnitus and hyperacusis in animals. Front Neurol 5, 179.

Henry, J.A., 2016. "measurement” of Tinnitus. Otol Neurotol 37, e276-e285.

Henry, J.A., Dennis, K.C., Schechter, M.A., 2005. General review of tinnitus: Prevalence, mechanisms, effects, and management. J. Speech Lang. Hear. Res.

Ison, J.R., 1982. Temporal acuity in auditory function in the rat: Reflex inhibition by brief gaps in noise. J. Comp. Physiol. Psychol. 96, 945-954.

Jafari, Z., Kolb, B.E., Mohajerani, M.H., 2019. Age-related hearing loss and tinnitus, dementia risk, and auditory amplification outcomes. Ageing Res. Rev.

Janssen, T., Boege, P., Oestreicher, E., Arnold, W., 2000. Tinnitus and 2f1-f2 distortion product otoacoustic emissions following salicylate overdose. J. Acoust. Soc. Am. 107, 1790-1792.

Jastreboff, P.J., 1990. Phantom auditory perception (tinnitus): mechanisms of generation and perception. Neurosci. Res.

Kaltenbach, J.A., Zacharek, M.A., Zhang, J., Frederick, S., 2004. Activity in the dorsal cochlear nucleus of hamsters previously tested for tinnitus following intense tone exposure. Neurosci. Lett. 355, 121- 
125.

Kim, M.J., Park, S.Y., Park, J.M., Yu, H.J., Park, I., Park, S.N., 2021. Evidence of Tinnitus Development Due to Stress: An Experimental Study in Rats. Laryngoscope.

Knipper, M., van Dijk, P., Schulze, H., Mazurek, B., Krauss, P., Scheper, V., Warnecke, A., Schlee, W., Schwabe, K., Singer, W., Braun, C., Delano, P.H., Fallgatter, A.J., Ehlis, A.C., Searchfield, G.D., Munk, M.H.J., Baguley, D.M., Rüttiger, L., 2020. The neural bases of tinnitus: lessons from deafness and cochlear implants. J. Neurosci.

Koch, M., 1999. The neurobiology of startle. Prog. Neurobiol.

Ku, Y., Ahn, J. woo, Kwon, C., Kim, D.Y., Suh, M.-W., Park, M.K., Lee, J.H., Oh, S.H., Kim, H.C., 2017. The gap-prepulse inhibition deficit of the cortical N1-P2 complex in patients with tinnitus: The effect of gap duration. Hear. Res. 348, 120-128.

Lanaia, V., Tziridis, K., Schulze, H., 2021. Salicylate-Induced Changes in Hearing Thresholds in Mongolian Gerbils Are Correlated With Tinnitus Frequency but Not With Tinnitus Strength. Front. Behav. Neurosci. 15.

Landgrebe, M., Langguth, B., Rosengarth, K., Braun, S., Koch, A., Kleinjung, T., May, A., de Ridder, D., Hajak, G., 2009. Structural brain changes in tinnitus: Grey matter decrease in auditory and nonauditory brain areas. NeuroImage 46, 213-218.

Lissek, S., Baas, J.M.P., Pine, D.S., Orme, K., Dvir, S., Nugent, M., Rosenberger, E., Rawson, E., Grillon, C., 2005. Airpuff startle probes: An efficacious and less aversive alternative to white-noise. Biol. Psychol. 68, 283-297.

Liu, X.P., Chen, L., 2015. Forward acoustic masking enhances the auditory brainstem response in a diotic, but not dichotic, paradigm in salicylate-induced tinnitus. Hear. Res. 323, 51-60.

Lobarinas, E., Hayes, S.H., Allman, B.L., 2013. The gap-startle paradigm for tinnitus screening in animal 
models: Limitations and optimization. Hear. Res. 295, 150-160.

Lockwood, A.H., Salvi, R.J., Burkard, R.F., 2002. Tinnitus. N. Engl. J. Med. 347, 904-910.

Longenecker, R.J., Galazyuk, A. V., 2012. Methodological optimization of tinnitus assessment using prepulse inhibition of the acoustic startle reflex. Brain Res. 1485, 54-62.

Longenecker, R.J., Galazyuk, A. V., 2011. Development of tinnitus in CBA/CaJ mice following sound exposure. J. Assoc. Res. Otolaryngol. 12, 647-658.

Longenecker, R.J., Kristaponyte, I., Nelson, G.L., Young, J.W., Galazyuk, A. V., 2018. Addressing variability in the acoustic startle reflex for accurate gap detection assessment. Hear. Res. 363, 119135.

Magliulo, G., Zardo, F., D’Amico, R., Varacalli, S., Forino, M., 2000. Acoustic neuroma: Postoperative quality of life. J. Otol. 29, 344-347.

Mahmoudian, S., Farhadi, M., Najafi-Koopaie, M., Darestani-Farahani, E., Mohebbi, M., Dengler, R., Esser, K.H., Sadjedi, H., Salamat, B., Danesh, A.A., Lenarz, T., 2013. Central auditory processing during chronic tinnitus as indexed by topographical maps of the mismatch negativity obtained with the multi-feature paradigm. Brain Res. 1527, 161-173.

Martel, D.T., Pardo-Garcia, T.R., Shore, S.E., 2019. Dorsal Cochlear Nucleus Fusiform-cell Plasticity is Altered in Salicylate-induced Tinnitus. Neuroscience 407, 170-181.

Mccabe, P.A., Dey, F.L., 1965. XXVIII: The effect of aspirin upon auditory sensitivity. Ann. Otol. Rhinol. Laryngol. 74, 312-325.

Mennink, L.M., Van Dijk, J.M.C., Van Der Laan, B.F.A.M., Metzemaekers, J.D.M., Van Laar, P.J., Van Dijk, P., 2018. The relation between flocculus volume and tinnitus after cerebellopontine angle tumor surgery. Hear. Res. 361, 113-120. 
Miwa, H., Nohara, C., Hotta, M., Shimo, Y., Amemiya, K., 1998. Somatosensory-evoked blink response: Investigation of the physiological mechanism. Brain 121, 281-291.

Mühlau, M., Rauschecker, J.P., Oestreicher, E., Gaser, C., Röttinger, M., Wohlschläger, A.M., Simon, F., Etgen, T., Conrad, B., Sander, D., 2006. Structural brain changes in tinnitus. Cereb. Cortex 16, $1283-1288$.

Mühlnickel, W., Elbert, T., Taub, E., Flor, H., 1998. Reorganization of auditory cortex in tinnitus. Proc. Natl. Acad. Sci. 95, 10340-10343.

Neff, P., Simões, J., Psatha, S., Nyamaa, A., Boecking, B., Rausch, L., Dettling-Papargyris, J., Funk, C., Brueggemann, P., Mazurek, B., 2021. The impact of tinnitus distress on cognition. Scientific Reports 11, 2243.

Newman, C.W., Jacobson, G.P., Spitzer, J.B., 1996. Development of the tinnitus handicap inventory. Arch. Otolaryngol. - Head Neck Surg. 122, 143-148.

Ng, C.W., Navarro, X., Engle, J.R., Recanzone, G.H., 2015. Age-related changes of auditory brainstem responses in nonhuman primates. J. Neurophysiol. 114, 455-467.

Noreña, A.J., 2011. An integrative model of tinnitus based on a central gain controlling neural sensitivity. Neurosci. Biobehav. Rev.

Noreña, A.J., Eggermont, J.J., 2003. Changes in spontaneous neural activity immediately after an acoustic trauma: Implications for neural correlates of tinnitus. Hear. Res. 183, 137-153.

Öngür, D., Price, J.L., 2000. The organization of networks within the orbital and medial prefrontal cortex of rats, monkeys and humans. Cereb. Cortex.

Ornitz, E.M., Guthrie, D., Kaplan, A.R., Lane, S.J., Norman, R.J., 1986. Maturation of startle modulation. Psychophysiology 23, 624-634. 
Paraskevopoulos, E., Dobel, C., Wollbrink, A., Salvari, V., Bamidis, P.D., Pantev, C., 2019. Maladaptive alterations of resting state cortical network in Tinnitus: A directed functional connectivity analysis of a larger MEG data set. Sci. Rep. 9.

Preuss, T.M., 1995. Do rats have prefrontal cortex? The Rose-Woolsey-Akert program reconsidered. J Cogn Neurosci.

Rauschecker, J.P., 1999. Auditory cortical plasticity: A comparison with other sensory systems. Trends Neurosci.

Rauschecker, J.P., Leaver, A.M., Mühlau, M., 2011. Tuning Out the Noise: Limbic-Auditory Interactions in Tinnitus. Neuron

Rauschecker, J.P., May, E.S., Maudoux, A., Ploner, M., 2016. Frontostriatal Gating of Tinnitus and Chronic Pain. Trends Cog. Sci.

Recanzone, G., 2018. The effects of aging on auditory cortical function. Hear. Res. https://doi.org/10.1016/j.heares.2018.05.013

Reed, G.F., 1960. An audiometric study of two hundred cases of subjective tinnitus. AMA Arch Otolaryngol. 71, 84-94.

Roeser, R.J., Price, D.R., 1980. Clinical experience with tinnitus maskers. Ear Hear. 1, 63-68.

Salazar, J.W., Meisel, K., Smith, E.R., Quiggle, A., McCoy, D.B., Amans, M.R., 2019. Depression in patients with tinnitus: A systematic review. Otolaryngol. Head Neck Surg.

Schecklmann, M., Landgrebe, M., Poeppl, T.B., Kreuzer, P., Männer, P., Marienhagen, J., Wack, D.S., Kleinjung, T., Hajak, G., Langguth, B., 2013. Neural correlates of tinnitus duration and Distress: A positron emission tomography study. Hum. Brain Mapp. 34, 233-240.

Schmidt, S.A., Zimmerman, B., Bido Medina, R.O., Carpenter-Thompson, J.R., Husain, F.T., 2018. 
Changes in gray and white matter in subgroups within the tinnitus population. Brain Res. 1679, 6474.

Schmitt, C., Patak, M., Kroner-Herwig, B., 2000. Stress and the onset of sudden hearing loss and tinnitus. Int. Tinnitus J. 6, 41-49.

Seydell-Greenwald, A., Leaver, A.M., Turesky, T.K., Morgan, S., Kim, H.J., Rauschecker, J.P., 2012. Functional MRI evidence for a role of ventral prefrontal cortex in tinnitus. Brain Research 1485, $22-$ 39.

Shargorodsky, J., Curhan, G.C., Farwell, W.R., 2010. Prevalence and characteristics of tinnitus among US adults. Am. J. Med. 123, 711-718.

Shore, S.E., Roberts, L.E., Langguth, B., 2016. Maladaptive plasticity in tinnitus-triggers, mechanisms and treatment. Nat. Rev. Neurol.

Shore, S.E., Wu, C., 2019. Mechanisms of Noise-Induced Tinnitus: Insights from Cellular Studies. Neuron.

Singer, W., Zuccotti, A., Jaumann, M., Lee, S.C., Panford-Walsh, R., Xiong, H., Zimmermann, U., Franz, C., Geisler, H.S., Köpschall, I., Rohbock, K., Varakina, K., Verpoorten, S., Reinbothe, T., Schimmang, T., Rüttiger, L., Knipper, M., 2013. Noise-induced inner hair cell ribbon loss disturbs central arc mobilization: A novel molecular paradigm for understanding tinnitus. Mol. Neurobiol.

Stapells, D.R., Oates, P., 1997. Estimation of the pure-tone audiogram by the auditory brainstem response: a review. Audiology \& neuro-otology 2, 257-80.

Stolzberg, D., Chen, G.D., Allman, B.L., Salvi, R.J., 2011. Salicylate-induced peripheral auditory changes and tonotopic reorganization of auditory cortex. Neuroscience 180, 157-164.

Stolzberg, D., Salvi, R.J., Allman, B.L., 2012. Salicylate toxicity model of tinnitus. Front. Syst. Neurosci. 
Swerdlow, N.R., Braff, D.L., Geyer, M.A., 1999. Cross-species studies of sensorimotor gating of the startle reflex, in: Ann. N. Y. Acad. Sci. pp. 202-216.

Szibor, A., Mäkitie, A., Aarnisalo, A.A., 2019. Tinnitus and suicide: An unresolved relation. Audiol Res. $9,10-13$.

Turner, J.G., Brozoski, T.J., Bauer, C.A., Parrish, J.L., Myers, K., Hughes, L.F., Caspary, D.M., 2006. Gap detection deficits in rats with tinnitus: A potential novel screening tool. Behav. Neurosci. 120, $188-195$.

Valls-Sole, J., 2012. Assessment of excitability in brainstem circuits mediating the blink reflex and the startle reaction. Clin Neurophysiol.

Vanneste, S., Plazier, M., der Loo, E. van, de Heyning, P. Van, Congedo, M., De Ridder, D., 2010. The neural correlates of tinnitus-related distress. NeuroImage 52, 470-480.

von der Behrens, W., 2014. Animal models of subjective tinnitus. Neural Plast.

Wienbruch, C., Paul, I., Weisz, N., Elbert, T., Roberts, L.E., 2006. Frequency organization of the 40-Hz auditory steady-state response in normal hearing and in tinnitus. NeuroImage 33, 180-194.

Yang, G., Lobarinas, E., Zhang, L., Turner, J., Stolzberg, D., Salvi, R., Sun, W., 2007. Salicylate induced tinnitus: Behavioral measures and neural activity in auditory cortex of awake rats. Hear. Res. 226, 244-253. 
Table 1

Tinnitus characteristics.

\begin{tabular}{|c|c|c|c|c|c|c|c|c|}
\hline $\begin{array}{c}\text { Patient } \\
\#\end{array}$ & $\begin{array}{c}\text { Age } \\
\text { (years) }\end{array}$ & $\begin{array}{c}\text { Associated } \\
\text { aetiology }\end{array}$ & Description & Location & $\begin{array}{c}\text { Frequency } \\
(\mathrm{kHz})\end{array}$ & $\begin{array}{c}\text { Loudness } \\
\text { (dB SL) }\end{array}$ & $\begin{array}{c}\text { Onset } \\
\text { (years ago) }\end{array}$ & $\begin{array}{c}\text { Impact } \\
\text { (THI scores) }\end{array}$ \\
\hline 1 & 69 & Chemotherapy & Tone & Both ears & 9 & 2 & $>10$ & 4 \\
\hline 2 & 50 & Unknown & Tone & $\begin{array}{l}\text { Both ears, } \\
\text { worse in } \\
\text { right }\end{array}$ & 8 & 18 & 19 & 14 \\
\hline 3 & 46 & Stress & Noise & $\begin{array}{l}\text { Inside the } \\
\text { head }\end{array}$ & 8 & 4 & 5 & 86 \\
\hline 4 & 39 & Loud blast & Tone & Both ears & 9 & 18 & 19 & 4 \\
\hline 5 & 55 & Loud blast & Crickets & Both ears & 10 & 10 & 2 & 48 \\
\hline 6 & 40 & $\begin{array}{l}\text { Prolonged } \\
\text { sound } \\
\text { exposure }\end{array}$ & Tone & $\begin{array}{c}\text { Both ears, } \\
\text { worse in } \\
\text { left }\end{array}$ & 8 & 12 & 10 & 6 \\
\hline 7 & 20 & Labyrinthitis & Noise & Left ear & 8 & 10 & 5 & 46 \\
\hline 8 & 75 & Unknown & $\begin{array}{l}\text { Hissing, } \\
\text { humming, } \\
\text { whooshing }\end{array}$ & Both ears & 8 & 8 & 5 & 4 \\
\hline 9 & 55 & $\begin{array}{l}\text { Unknown } \\
\text { Sound }\end{array}$ & Tone & Both ears & 8 & 4 & $?$ & 10 \\
\hline 10 & 27 & exposure & tone & Both ears & 6 & 20 & $>9$ & 6 \\
\hline 11 & 63 & Loud blast & tone & Both ears & 14 & 6 & 30 & 8 \\
\hline
\end{tabular}


Table 2

ANOVA results from both NHPs (monkey C and S).

\begin{tabular}{|c|c|c|c|c|c|c|}
\hline & \multicolumn{2}{|c|}{$F$-value } & \multicolumn{2}{|c|}{$P$-value } & \multicolumn{2}{|c|}{ Effect size $\left(\eta^{2} p\right)$} \\
\hline & $\mathrm{C}$ & S & $\mathrm{C}$ & S & $\mathrm{C}$ & $\mathrm{S}$ \\
\hline \multicolumn{7}{|l|}{$1 \mathrm{kHz}$} \\
\hline Recording block & 1.31 & 0.23 & 0.31 & 0.80 & 0.21 & 0.05 \\
\hline Intensity & 21.34 & 19.53 & $2.4 \times 10^{-4}$ & $3.5 \times 10^{-4}$ & 0.81 & 0.80 \\
\hline Interaction & 0.31 & 0.35 & 0.87 & 0.84 & 0.06 & 0.07 \\
\hline \multicolumn{7}{|l|}{$2 \mathrm{kHz}$} \\
\hline Recording block & 3.96 & 1.39 & 0.05 & 0.29 & 0.44 & 0.22 \\
\hline Intensity & 50.25 & 29.22 & $6 \times 10^{-6}$ & $6.7 \times 10^{-5}$ & 0.91 & 0.85 \\
\hline Interaction & 2.96 & 0.27 & 0.06 & 0.89 & 0.35 & 0.52 \\
\hline \multicolumn{7}{|l|}{$4 \mathrm{kHz}$} \\
\hline Recording block & 3.67 & 0.20 & 0.08 & 0.82 & 0.40 & 0.04 \\
\hline Intensity & 26.74 & 35.12 & $9.7 \times 10^{-5}$ & $3.0 \times 10^{-5}$ & 0.84 & 0.86 \\
\hline Interaction & 1.43 & 0.68 & 0.26 & 0.62 & 0.22 & 0.12 \\
\hline \multicolumn{7}{|l|}{$8 \mathrm{kHz}$} \\
\hline Recording block & 4.40 & 0.85 & 0.04 & 0.46 & 0.47 & 0.15 \\
\hline Intensity & 25.57 & 114.05 & $1.2 \times 10^{-4}$ & $1.3 \times 10^{-7}$ & 0.84 & 0.96 \\
\hline Interaction & 0.39 & 0.69 & 0.81 & 0.61 & 0.07 & 0.12 \\
\hline \multicolumn{7}{|l|}{$16 \mathrm{kHz}$} \\
\hline Recording block & 12.46 & 1.09 & $1.9 \times 10^{-3}$ & 0.37 & 0.71 & 0.18 \\
\hline Intensity & 14.90 & 26.06 & $1.0 \times 10^{-3}$ & $1.9 \times 10^{-4}$ & 0.75 & 0.84 \\
\hline Interaction & 5.38 & 3.12 & $4.17 \times 10^{-3}$ & 0.04 & 0.52 & 0.39 \\
\hline
\end{tabular}

The $p$-values of significant interaction effects are highlighted in bold. 
Table 3

ANOVA results from humans.

\begin{tabular}{cccc}
\hline & $F$-value & $P$-value & Effect size $\left(\eta^{2} p\right)$ \\
\cline { 2 - 4 } $1 \mathrm{kHz}$ & & & \\
Group & $5.1 \times 10^{-4}$ & 0.98 & $2.6 \times 10^{-5}$ \\
Intensity & 29.87 & $5.1 \times 10^{-8}$ & 0.60 \\
Interaction & 0.28 & 0.76 & 0.01 \\
$2 \mathrm{kHz}$ & & & \\
Group & 0.57 & 0.46 & 0.03 \\
Intensity & 13.22 & $3.9 \times 10^{-5}$ & 0.40 \\
Interaction & 0.76 & 0.47 & 0.04 \\
$4 \mathrm{kHz}$ & & & \\
Group & 2.10 & 0.16 & 0.10 \\
Intensity & 21.83 & $3.9 \times 10^{-7}$ & 0.52 \\
Interaction & 0.34 & 0.72 & 0.17 \\
$8 \mathrm{kHz}$ & & & \\
Group & 8.68 & $8.0 \times 10^{-3}$ & 0.30 \\
Intensity & 21.37 & $4.9 \times 10^{-7}$ & 0.52 \\
Interaction & 7.29 & $\mathbf{2 . 0} \times \mathbf{1 0}^{-3}$ & 0.27 \\
$16 \mathrm{kHz}$ & & & \\
Group & 1.29 & 0.27 & 0.06 \\
Intensity & 23.23 & $2.0 \times 10^{-7}$ & 0.54 \\
Interaction & 0.68 & 0.52 & 0.03 \\
\hline
\end{tabular}

The $p$-values of significant interaction effects are highlighted in bold. 

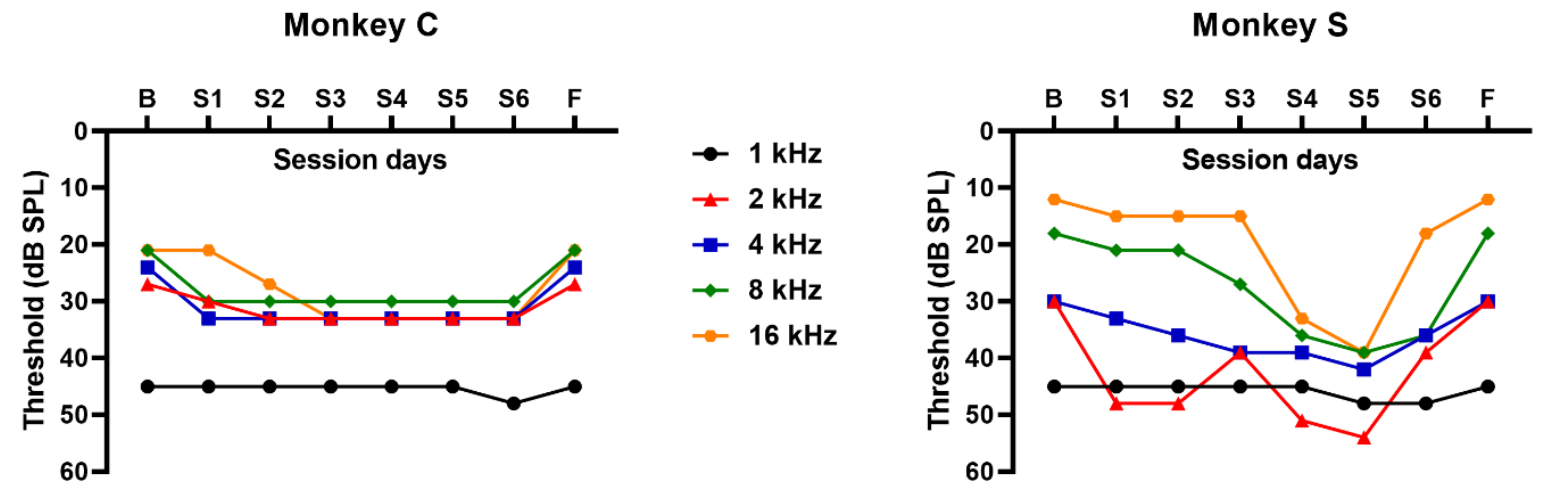

Fig. 1. Audiograms of both NHPs (C and S) revealed by ABR recordings across session days. S1-S6 indicate days with salicylate administration. Values across the particular Baseline (B) and Follow-up (F) days were identical; thus collapsed here. 


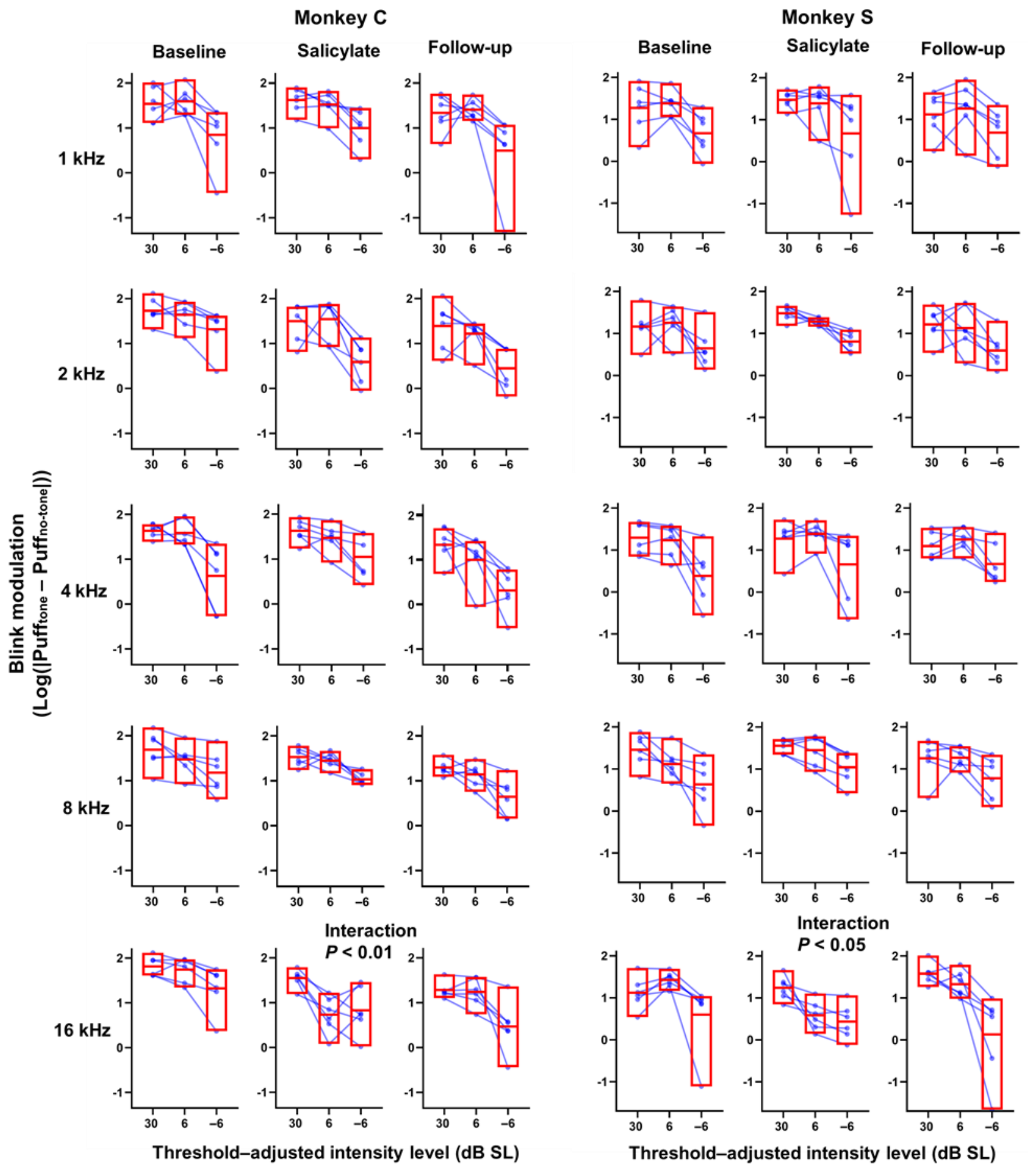

Fig.2 Blink modulation scores of both NHPs (monkey C and S) across all conditions of frequency, intensity and recording block. The floating bars represent the range and the within-lines the mean. The blue connected dots represent the individual values of the session days $(N=6)$. Significant interaction effects are indicated. 
Patients

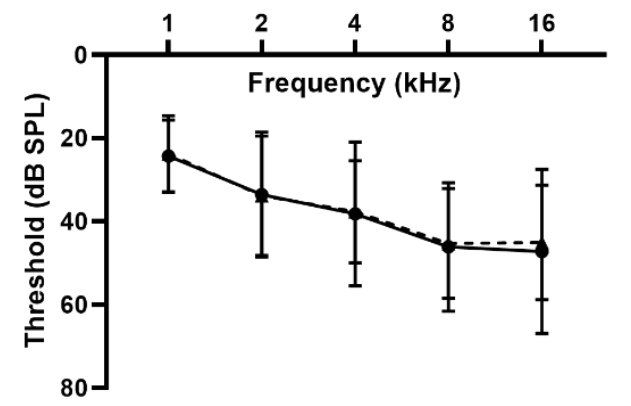

Controls

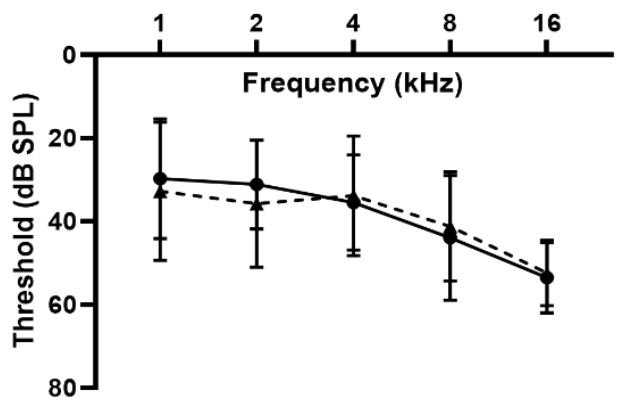

Fig. 3 Audiograms of both groups (patients and controls) revealed by ABR recordings and behavioral testing. Plotted are the means together with the standard deviations as whiskers. 


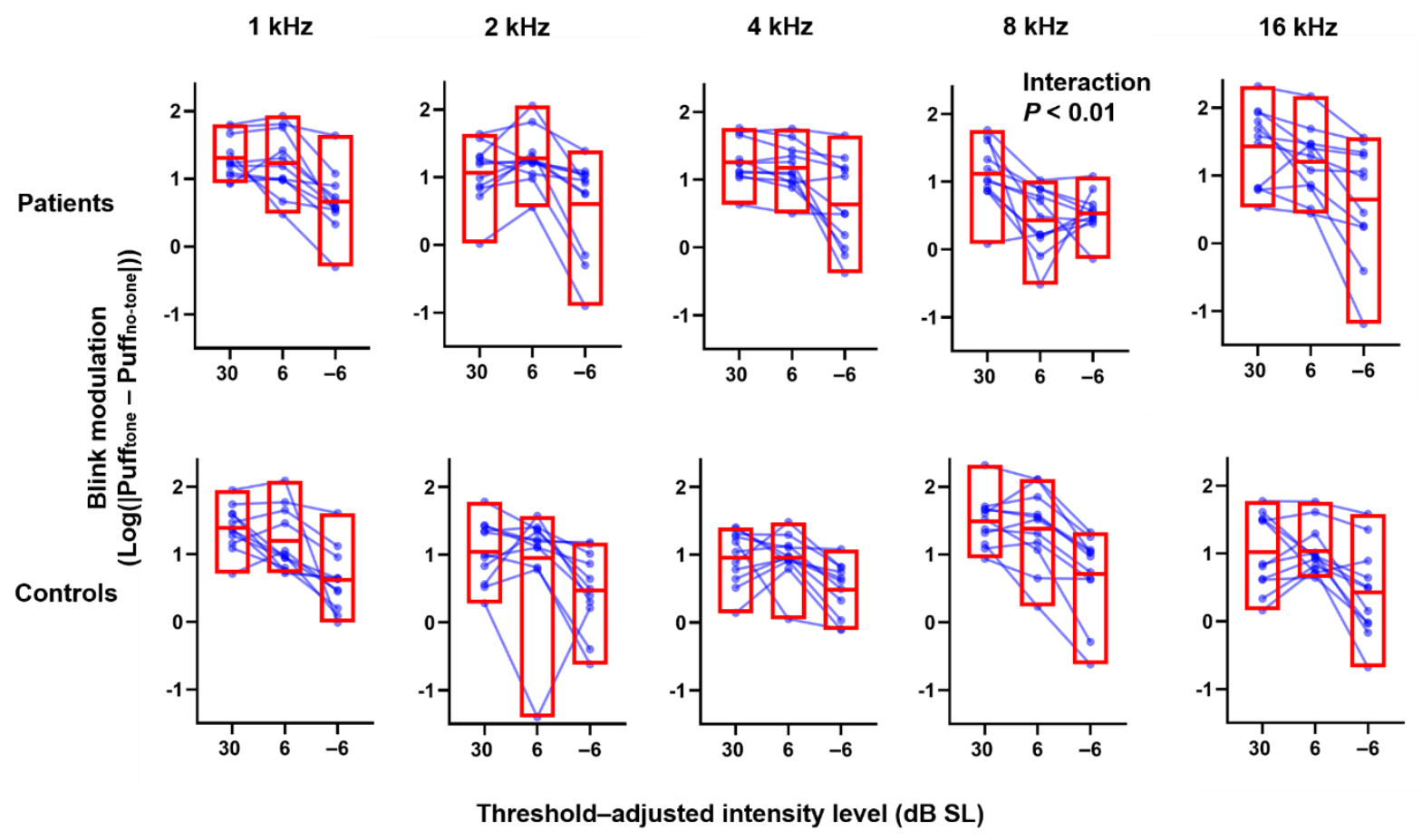

Fig. 4 Blink modulation scores of both groups (patients and controls) across all conditions of frequency and intensity. The floating bars represent the range and the within-lines the mean. The blue connected dots represent the individual values of the participants $(N=11)$. The significant interaction effect is indicated. 


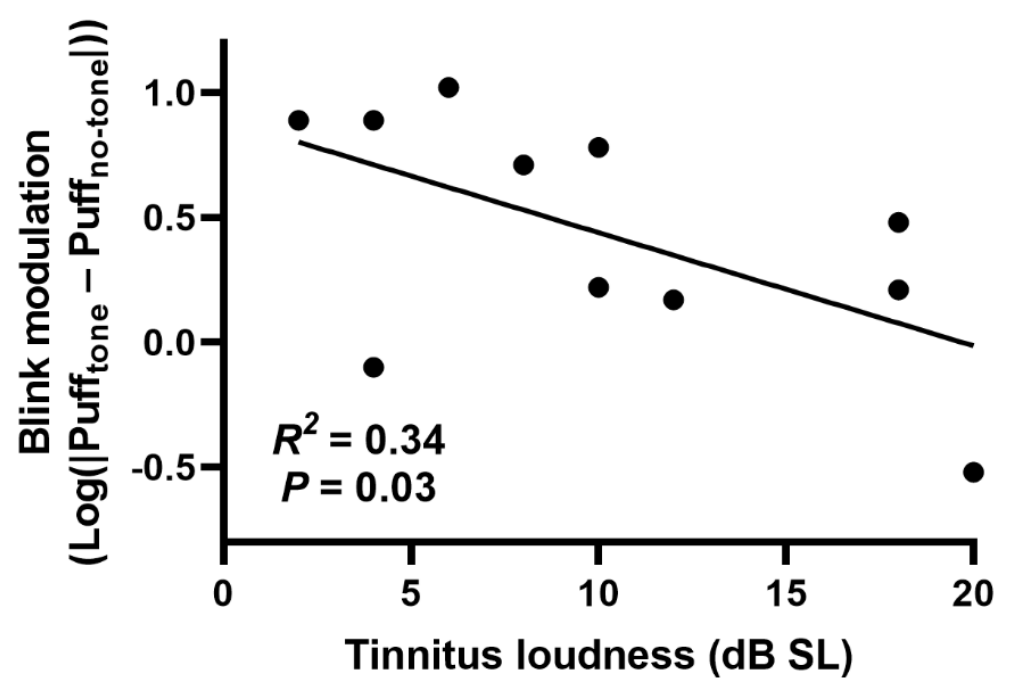

Fig. 5 Relationship between tinnitus loudness and the blink modulation scores in response to the $8 \mathrm{kHz}$-tone set at 6 $\mathrm{dB}$ SL. The dots depict the individual pairs of values revealed by each patient $(N=11)$. Tinnitus loudness was independently determined by audiologists. 

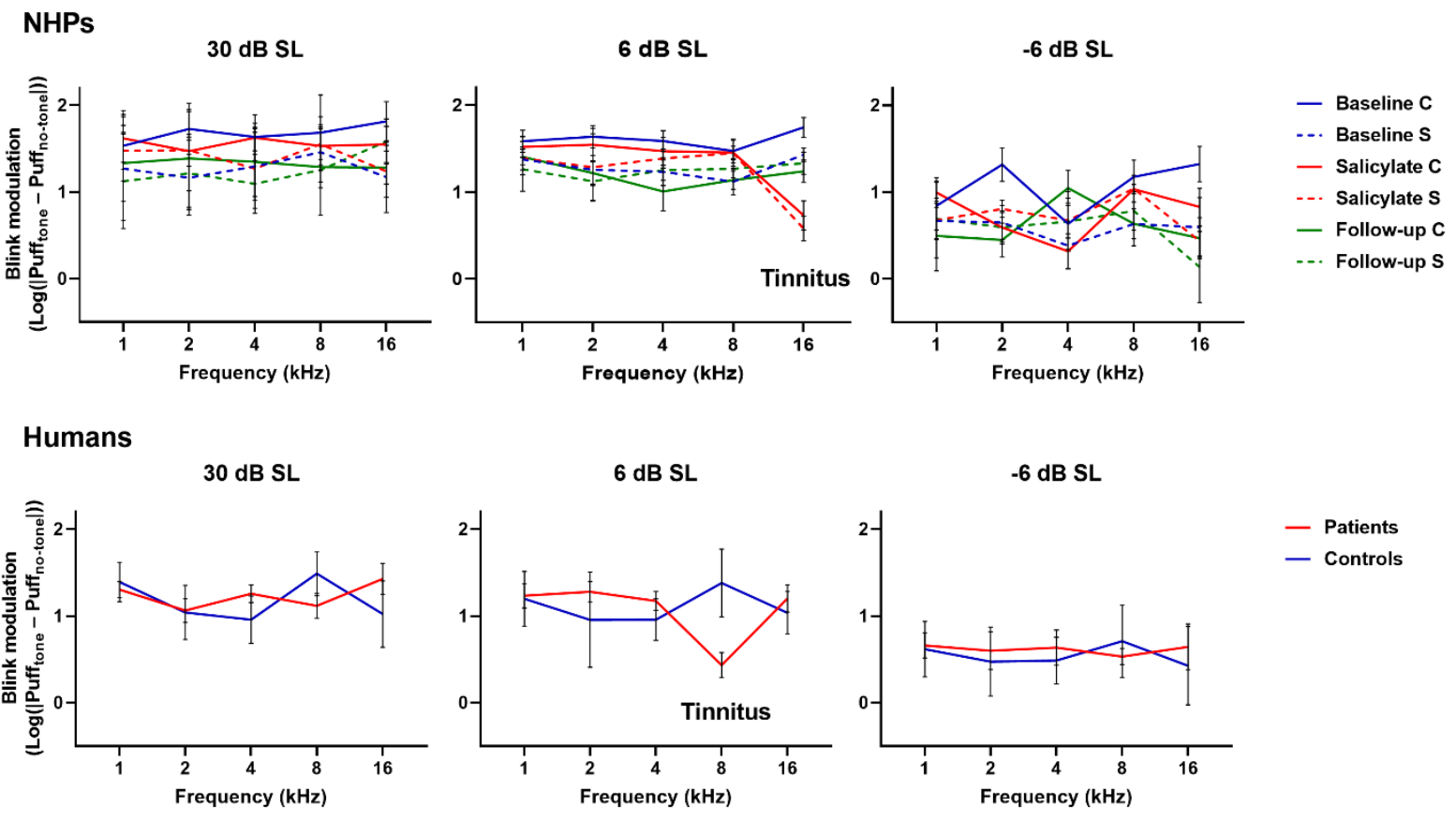

Fig. 6 Summary of the blink modulation findings. On top, the means of the blink modulation scores from both NHPs (C and S) are shown across all conditions of frequency, intensity and recording block. Below, the means of the blink modulation scores from both groups (patients and controls) are shown across all conditions of frequency and intensity. The whiskers depict standard error bars. Tinnitus effects are indicated. 